\title{
Assessment of Knowledge, Attitude, and Practice of Iranian Nurses towards Toothbrush Maintenance and Use
}

\author{
Mohammad Nazarianpirdosti, ${ }^{1}$ Maryam Janatolmakan $\left(\mathbb{D},{ }^{2}\right.$ Bahare Andayeshgar, ${ }^{3}$ \\ and Alireza khatony $\mathbb{D}^{2,4}$ \\ ${ }^{1}$ Student Research Committee, Kermanshah University of Medical Sciences, Kermanshah, Iran \\ ${ }^{2}$ Social Development and Health Promotion Research Centre, Health Institute, Kermanshah University of Medical Sciences, \\ Kermanshah, Iran \\ ${ }^{3}$ School of Health, Kermanshah University of Medical Sciences, Kermanshah, Iran \\ ${ }^{4}$ Infectious Diseases Research Center, Kermanshah University of Medical Sciences, Kermanshah, Iran \\ Correspondence should be addressed to Alireza khatony; akhatony@gmail.com
}

Received 3 October 2021; Accepted 3 December 2021; Published 14 December 2021

Academic Editor: Maria H F Grypdonck

Copyright ( 2021 Mohammad Nazarianpirdosti et al. This is an open access article distributed under the Creative Commons Attribution License, which permits unrestricted use, distribution, and reproduction in any medium, provided the original work is properly cited.

\begin{abstract}
Background. Since nurses are considered a role model in society, they should have sufficient knowledge, attitude, and practice in the field of oral hygiene. This study was aimed to assess the nurses' knowledge, practice, and attitude towards toothbrush maintenance and use. Methods. In this cross-sectional study, 325 nurses working in hospitals affiliated to Kermanshah University of Medical Sciences were randomly recruited. Data collection tools included a demographic information form and a researchermade questionnaire on knowledge, attitude, and practice regarding toothbrush maintenance and use. Data were analyzed by SPSS software using descriptive and inferential statistics (Mann-Whitney $U$ and Kruskal-Wallis H). Results. The mean scores of nurses' knowledge, attitude, and practice were 59.2 $\pm 16.4,64.2 \pm 20.6$, and $51.4 \pm 17.0$ out of 100, respectively. There was no statistically significant relation between nurses' knowledge, attitude, and practice and their gender, age, level of education, and work experience. Conclusions. Nurses had moderate knowledge, attitude, and practice regarding toothbrush maintenance and use, which is not very desirable given their role model. Therefore, training courses are recommended to be held to increase the nurses' knowledge, attitude, and practice regarding toothbrush maintenance and use.
\end{abstract}

\section{Background}

Brushing is one of the most important and effective self-care methods which prevents oral diseases [1-3]. In addition, brushing reduces dental plaque and thus prevents decay and related diseases [4-8]. Therefore, it is highly important for nurses to have adequate knowledge about the correct brushing method and also toothbrush maintenance, replacement, and cleaning [9-12]. If the toothbrush is not properly maintained and used, it can cause oral infections and diseases [9-15]. Changing the toothbrush every 2.5-6 months and brushing for two minutes or more twice a day are some correct methods of toothbrush use [13, 16-22].

Today, as the largest group in the health sector, nurses have an important role in promoting health policies in the field of oral health [23-27]. Therefore, adequate knowledge and practice and positive attitude regarding toothbrush maintenance and use is of special importance for nurses [28-32]. Surveys in Europe and the United States show that nurses consider oral health one of the most important nursing practices [1,33]. A study in Malaysia showed that despite the limited knowledge of nurses about oral health, they had a good attitude to it [34]. The results of a study in India (2018) on oral health showed that $70 \%$ of nurses had poor knowledge, $83 \%$ had a positive attitude, and $69 \%$ had poor practice [30]. A study also showed that $82 \%$ of Iranian nurses had poor oral care practice [35]. The results of a study in Norway (2012) showed that $80 \%$ of nurses considered oral health an important issue, while $9.1 \%$ found patient oral care unpleasant [36]. In another study, the knowledge of Nigerian 
nurses and midwives regarding oral health was inadequate [37]. The results of a study on Australian nurses showed that $74.0 \%$ of them were aware of the important oral health practices [38].

Considering the educational role of nurses and the lack of information about the knowledge, attitude, and practice of the nurses of Kermanshah University of Medical Sciences regarding toothbrush maintenance and use, the current study was conducted to shed more light on this lacuna. This study sought to answer the following questions:

(1) What is the nurses' level of knowledge about toothbrush maintenance and use?

(2) What is the nurses' attitude and practice regarding toothbrush maintenance and use?

(3) What is the relationship between nurses' knowledge, attitude, and practice in toothbrush maintenance and use and their demographic variables?

\section{Materials and Methods}

2.1. Study Design. The present descriptive-analytical crosssectional study was conducted from March to May 2019. The study was performed based on STROBE reporting criteria.

2.2. Sample and Sampling Method. The study population $(n=2042)$ consisted of nurses working in hospitals affiliated to Kermanshah University of Medical Sciences (7 hospitals). The sample size was estimated to be 325 using Cochran's formula and the results of the study of Sharif et al. with $95 \%$ confidence and the first type error equal to 5\% [34]. The inclusion criteria consisted of employment in the field of nursing and consent to participation in the study.

2.3. Instruments. The study tools included a personal information form and a questionnaire on nurses' knowledge, attitude, and practice in toothbrush maintenance and use. The personal information form included 5 questions on gender, age, level of education, marital status, and work experience.

A valid and reliable questionnaire was used to assess the nurses' knowledge, attitude, and practice in toothbrush maintenance and use. This questionnaire was developed and validated by Janatolmakan et al. and had good psychometric properties. They examined the validity of the questionnaire by the qualitative and quantitative content validity method. In the qualitative section, the judgment of experts has been used, and in the quantitative section, the content validity index has been calculated, which has been equal to $0.87,0.89$, and 0.88 for the sections of knowledge, attitude, and practice, respectively. The reliability of the instrument was also tested and confirmed by the test-retest method. The correlation coefficients for the scales of knowledge, attitude, and practice were $0.87,0.88$, and 0.86 , respectively [39].

The first part of the questionnaire was allocated to the assessment of knowledge and consisted of 10 multiple choice questions. Some of the questions in this section were as follows: "What is the right water temperature for brushing?"
"What is the best way to brush?" and "When should the toothbrush be washed?"

To score this section, the correct and incorrect answers were given a score of one and zero, respectively. The range of scores was between 0 and 10 , which was calculated on the basis of 100 and was divided as poor $(\leq 49)$, medium (50-74), and good $(\geq 75)$ knowledge.

The second part, with 6 questions, was allocated to evaluate the nurses' attitudes toward toothbrush maintenance and use. The items in this section were of two-choice type, and the answers included "agree and disagree." Two of the items in this section were "The harder the toothbrush material, the better its function" and "foreign toothbrushes are more durable." To calculate the scores, the answers "I agree" and "I disagree" were given one and zero points, respectively. The range of scores was between 0 and 6 , which was calculated on the basis of 100 and was divided as negative $(\leq 49)$ and positive $(\geq 50)$ attitude.

The third section consisted of 10 multiple-choice questions to evaluate the nurses' practice in toothbrush maintenance and use. Some of the questions in this section were "What type of toothbrush do you use?" "Where do you keep your toothbrush" and "When do you wash your toothbrush?" To calculate the score of this section, scores 1 and 0 were assigned to the correct and incorrect answers, respectively. The range of scores was between 0 and 10 , which was calculated on the basis of 100 and expressed as poor $(\leq 49)$, moderate $(50-74)$, and good $(\geq 75)$ practice.

2.4. Data Collection. After receiving the approval of the university ethics committee, the researcher attended the nurses' place of work according to the work schedule. First, the objectives of the study were explained to the nurses, and if they willing to participate in the study, the questionnaires were provided to them. To ensure the validity of the data, the participants were given enough time to complete the questionnaires.

2.5. Data Analysis. Data were analyzed by SPSS-16 software using descriptive and inferential statistics. Mean, standard deviation, median, and simple and relative frequency distributions were used for the descriptive statistics section. In the inferential statistics section, Mann-Whitney $U$, Kolmogorov-Smirnov, and Kruskal-Wallis tests were used. The Kolmogorov-Smirnov test was used to evaluate the normality of the distribution of knowledge, attitude, and practice variables. The results showed that these variables had an abnormal distribution. The Mann-Whitney $U$ test was used to examine the relationship between knowledge, attitude, and practice variables and gender and education level variables. The Kruskal-Wallis test was also used to examine the relationship between knowledge, attitude, and practice variables and age and work experience variables. The level of significance was set at $<0.05$.

2.6. Ethical Considerations. The Ethics Committee of Kermanshah University of Medical Sciences approved the study with the code IR.KUMS.REC.1397.874. Written 
informed consent was obtained from all participants. All participants were assured that their information and responses would be kept confidential. The protocol of the experiment was entirely in accordance to guidelines of national/international/institutional or Declaration of Helsinki.

\section{Results}

The mean age and work experience of the participants were $31.6 \pm 5.6$ and 5.3. \pm 2.7 years, respectively. Most of the participants were female $(n=197,60.4 \%)$, single $(n=179$, $55.1 \%)$, in the age range of $32-22(n=215,66.2 \%)$ and had a bachelor's degree $(n=299,92 \%)$ (Table 1$)$.

The mean score of nurses' knowledge about toothbrush maintenance and use was $59.2 \pm 16.4$ out of 100 . The mean scores of nurses' attitude and practice were $64.2 \pm 20.6$ and $51.4 \pm 17.0$ out of 100 , respectively (Figure 1 and Table 2 ). There was no statistically significant relationship between the mean scores of nurses' knowledge, attitude, and practice variables and gender, education, age, and work experience variables (Tables 3-5).

\section{Discussion}

This study aimed to investigate the Iranian nurses' knowledge, attitude, and practice regarding toothbrush maintenance and use. In the present study, most of the participants had a moderate level of knowledge and practice and an unfavorable attitude about the maintenance and use of toothbrushes. Proper maintenance and use of toothbrushes is an important part of oral hygiene [39]. Studies have reported that nurses have different levels of knowledge and practice about oral health. In this regard, Ibrahim et al. indicated that more than $90 \%$ of Sudanese nurses had good knowledge regarding oral health [2]. Furthermore, Sreenivasan et al. reported more than $80 \%$ of Indian nurses had good knowledge in this regard [33]. However, Ahmed et al. found 70\% of Indian nurses had poor knowledge about oral health [35]. In two studies conducted on the Indian and Iranian nurses, most of them had poor oral health practices $[25,35]$. In terms of attitude, Indian and Australian nurses have been found to have a favorable attitude in this regard $[35,38]$. It should be noted that differences in the demographic characteristics of the participants as well as the variety of data collection tools can make it difficult to compare the results of the studies. However, insufficient knowledge and practice of nurses about oral health especially toothbrush maintenance and use may be due to lack of continuous education. It seems that regular oral health training can improve nurses' awareness of their role as healthcare providers.

In the present study, no statistically significant relationship was found between age and nurses' knowledge, attitude, and practice. This finding is consistent with the findings of previous studies $[10,12,25,26,29,30,34,40]$. However, Lin et al. reported a statistically significant relationship between nurses' age and their oral care
TABle 1: Demographic characteristics of nurses.

\begin{tabular}{lcc}
\hline & Variables & $n(\%)$ \\
\hline \multirow{2}{*}{ Gender } & Male & $128(39.4)$ \\
& Female & $197(60.6)$ \\
Education & BSc. $^{*}$ & $299(92.0)$ \\
& MSc. $^{*}$ & $26(8.0)$ \\
Age (years) & $22-32$ & $193(59.4)$ \\
& $33-42$ & $124(38.2)$ \\
Work experience (years) & $43-52$ & $8(2.5)$ \\
& $1-9$ & $215(66.2)$ \\
& $10-18$ & $102(31.4)$ \\
& $19-28$ & $8(2.5)$ \\
\hline
\end{tabular}

${ }^{*}$ Bachelor of Science; ${ }^{*}$ Master of Science.

practice [32]. The principles of oral hygiene, especially brushing and toothbrush maintenance, should be considered a health measure from childhood and be continued throughout life.

Consistent with previous studies $[9-12,25]$, in the present study, no statistically significant relationship was found between gender and nurses' knowledge, practice, and attitude. However, Baseer et al. reported a significant relationship between gender and nurses' practice. Having sufficient knowledge and practice about oral health including toothbrush maintenance and use should be considered by both sexes.

In keeping with previous studies [9, 10, 12, 23-25, 29], no statistically significant relationship was observed between the level of education and nurses' knowledge, practice, and attitude. However, in some studies, a statistically significant relationship has been reported $[2,11]$. Nurses in every field of education are considered role models in society and should have a good attitude and sufficient knowledge and practice about oral health including toothbrush maintenance and use.

In the present study, there was no statistically significant relationship between work experience and the variables of knowledge, attitude, and practice of nurses. This finding is consistent with those of some previous studies $[2,23,29,33,34,40]$. Due to the nature of the nursing profession, nurses with any work experience are expected to have sufficient knowledge and practice and a favorable attitude towards the principles of maintenance and use of toothbrushes.

4.1. Study Limitations. This study faced three limitations. First, data were collected through a self-report method, which could have affected the accuracy of the results. However, the researcher tried to increase the validity of the results by reassuring the participants of anonymity of questionnaires and visiting them at an appropriate time to complete the questionnaires. Second, due to the cross-sectional nature of the study, it was not possible to determine the causal relationships between demographic and knowledge, attitude, and practice variables. Third, due to the fact that different tools with different cutting points have been used in different studies, it can limit the accurate comparison of study results. 


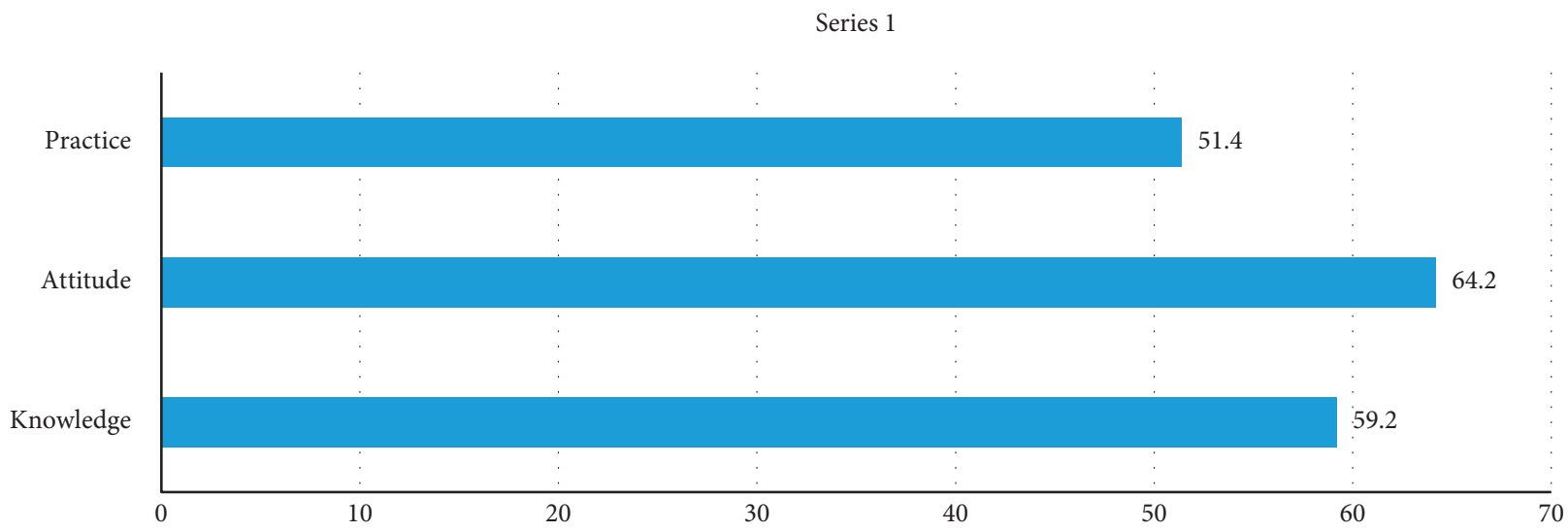

FIGURE 1: Comparison of nurses' knowledge, attitude and practice in toothbrush maintenance and use.

TABle 2: Nurses' knowledge, attitude, and practice scores in toothbrush maintenance and use.

\begin{tabular}{lcr}
\hline Variables & Median $\left(\mathrm{IQR}^{\ddagger}\right)$ & $\mathrm{Mean}^{ \pm} \mathrm{SD}^{\dagger}$ \\
\hline Knowledge & $60.0(20.0)$ & $59.3 \pm 16.4$ \\
Attitude & $66.7(33.3)$ & $64.2 \pm 20.7$ \\
Practice & $50.0(30.0)$ & $51.5 \pm 17.1$ \\
\hline
\end{tabular}

${ }^{\ddagger}$ Interquartile range; ${ }^{\dagger}$ standard deviation.

TABLE 3: Relationship between nurses' knowledge of toothbrush maintenance and use and demographic variables.

\begin{tabular}{|c|c|c|c|c|}
\hline & Variables & Median $\left(\mathrm{IQR}^{\ddagger}\right)$ & Mean $\pm \mathrm{SD}^{\dagger}$ & Test result \\
\hline \multirow{2}{*}{ Gender } & Male & $60(20.0)$ & $58.1(17.1)$ & $Z=-1.20$ \\
\hline & Female & $60(20.0)$ & $60.0(16.0)$ & $P=0.228$ \\
\hline \multirow{3}{*}{ Education } & BSc. $^{*}$ & $60(20.0)$ & $58.9(16.3)$ & $Z=-1.24$ \\
\hline & MSc.* & $60(30.0)$ & $63.8(17.9)$ & $P=0.213$ \\
\hline & $22-32$ & $60(20.0)$ & $58.9(16.3)$ & $X^{2}=1.47$ \\
\hline \multirow[t]{3}{*}{ Age (years) } & $33-42$ & $60(20.0)$ & $60.1(17.0)$ & $P=0.477$ \\
\hline & $43-52$ & $50(10.0)$ & $55.0(11.9)$ & \\
\hline & $1-9$ & $60(20.0)$ & $59.2(15.9)$ & $X^{2}=0.12$ \\
\hline \multirow[t]{2}{*}{ Work experience (years) } & $10-18$ & $60(20.0)$ & $59.4(17.9)$ & $P=0.942$ \\
\hline & $19-28$ & $55(17.5)$ & $58.7(11.2)$ & \\
\hline
\end{tabular}

*Interquartile range; ${ }^{\dagger}$ standard deviation; ${ }^{*}$ Bachelor of Science; * Master of Science.

TABLE 4: Relationship between nurses' attitude of toothbrush maintenance and use with demographic variables.

\begin{tabular}{|c|c|c|c|c|}
\hline & Variables & Median $\left(\mathrm{IQR}^{\ddagger}\right)$ & Mean $\pm \mathrm{SD}^{\dagger}$ & Test result \\
\hline \multirow{2}{*}{ Gender } & Male & $66.7(33.3)$ & $62.9(21.7)$ & $Z=-1.10$ \\
\hline & Female & $66.7(33.3)$ & $65.0(19.9)$ & $P=0.270$ \\
\hline \multirow{3}{*}{ Education } & BSc. $^{*}$ & $66.7(33.3)$ & $64.4(20.3)$ & $Z=-0.26$ \\
\hline & MSc. $^{*}$ & $66.7(33.3)$ & $61.5(24.8)$ & $P=0.791$ \\
\hline & $22-32$ & $66.7(25.0)$ & $63.5(20.5)$ & $X^{2}=0.90$ \\
\hline \multirow[t]{3}{*}{ Age (years) } & $33-42$ & $66.7(33.3)$ & $65.3(20.8)$ & $P=0.635$ \\
\hline & $43-52$ & $58.4(41.7)$ & $64.5(24.3)$ & \\
\hline & $1-9$ & $66.7(33.3)$ & $63.8(20.7)$ & $X^{2}=0.61$ \\
\hline \multirow[t]{2}{*}{ Work experience age (years) } & $10-18$ & $66.7(33.3)$ & $65.2(20.8)$ & $P=0.737$ \\
\hline & $19-28$ & $66.7(16.7)$ & $62.5(19.4)$ & \\
\hline
\end{tabular}

${ }^{\ddagger}$ Interquartile range; ${ }^{\dagger}$ standard deviation; ${ }^{\ddagger}$ Bachelor of Science; ${ }^{*}$ Master of Science. 
TABLE 5: Relationship between nurses' practice of toothbrush maintenance and use with demographic variables.

\begin{tabular}{|c|c|c|c|c|}
\hline & Variables & Median $\left(\mathrm{IQR}^{\ddagger}\right)$ & Mean $\pm \mathrm{SD}^{\dagger}$ & Test result \\
\hline \multirow{2}{*}{ Gender } & Male & $50(20.0)$ & $49.4(17.0)$ & $Z=-1.94$ \\
\hline & Female & $50(30.0)$ & $52.8(17.0)$ & $P=0.052$ \\
\hline \multirow{3}{*}{ Education } & BSc. $^{\ddagger}$ & $50(30.0)$ & $51.9(17.2)$ & $Z=-1.47$ \\
\hline & MSc.* & $40(32.5)$ & 46.9 (15.9) & $P=0.140$ \\
\hline & $22-23$ & $50(15.0)$ & $51.9(16.0)$ & $X^{2}=0.93$ \\
\hline \multirow[t]{3}{*}{ Age (years) } & $33-42$ & $50(30.0)$ & $50.9(18.7)$ & $P=0.626$ \\
\hline & $43-52$ & $40(20.0)$ & $47.5(18.3)$ & \\
\hline & $1-9$ & $50(20.0)$ & $51.5(15.9)$ & $X^{2}=0.34$ \\
\hline \multirow[t]{2}{*}{ Work experience (years) } & $10-18$ & $50(32.5)$ & $51.7(19.6)$ & $P=0.842$ \\
\hline & $19-28$ & $40(20.0)$ & $48.7(12.5)$ & \\
\hline
\end{tabular}

${ }^{\ddagger}$ Interquartile range; ${ }^{\dagger}$ standard deviation; ${ }^{*}$ Bachelor of Science; * Master of Science.

\section{Conclusion}

The results of this study indicated that nurses had moderate knowledge, attitude, and practice toward toothbrush maintenance and use, which does not seem acceptable. Since nurses are considered role models in society, they are required to have good knowledge, attitude, and practice regarding oral hygiene. Therefore, holding training courses on the principles of oral hygiene is recommended. It is also recommended to pay more attention to the issue of oral health, with emphasis on the maintenance and use of toothbrushes, in the nursing curriculum. Future studies are suggested to evaluate the factors related to nurses' knowledge, attitude, and practice regarding oral health and the effect of intervention measures on these variables.

\section{Data Availability}

The identified datasets analyzed during the current study are available from the corresponding author upon request.

\section{Ethical Approval}

The Ethics Committee of the Kermanshah University of Medical Sciences approved the study with the code IR.KUMS.REC.1397.874. The experiment protocol for involving humans was in accordance to guidelines of national/ international/institutional or Declaration of Helsinki.

\section{Consent}

The written informed consent was obtained from all the participants.

\section{Conflicts of Interest}

The authors declare that there are no conflicts of interest.

\section{Authors' Contributions}

MNP, MJ, BA, and AK designed the study and wrote the manuscript. MNP and MJ collected the data. BA analyzed the data. All the authors read and approved the version for submission.

\section{Acknowledgments}

The authors would like to thank all the nurses who participated in this study. The study was funded by Kermanshah University of Medical Sciences (97804).

\section{References}

[1] F. Mohamadkhah, F. Amin Shokravi, M. Karimy, and S. Faghihzadeh, "Effects of lecturing on selfcare oral health behaviors of elementary students," Medical Journal of the Islamic Republic of Iran, vol. 28, p. 86, 2014.

[2] S. M. Ibrahim, A. M. Mudawi, and O. Omer, "Nurses' knowledge, attitude and practice of oral care for intensive care unit patients," Open Journal of Stomatology, vol. 05, no. 07, pp. 179-186, 2015.

[3] H. Southern, "Oral care in cancer nursing: nurses' knowledge and education," Journal of Advanced Nursing, vol. 57, no. 6, pp. 631-638, 2007.

[4] L.-Y. Ju, S.-C. Weng, Y.-J. Chung et al., "Effects of the bass brushing method on dental plaque and pneumonia in older adults hospitalized with pneumonia after discharge: a randomized controlled trial," Applied Nursing Research, vol. 46, pp. 1-7, 2019.

[5] R. G. Ledder, J. Latimer, S. Forbes, J. L. Penney, P. K. Sreenivasan, and A. J. McBain, "Visualization and quantification of the oral hygiene effects of brushing, dentifrice use, and brush wear using a tooth brushing simulator," Frontiers in Public Health, vol. 7, p. 91, 2019.

[6] R. A. Ccahuana-Vasquez, R. Adam, E. Conde, J. M. Grender, P. Cunningham, and C. R. Goyal, "A 5-week randomized clinical evaluation of a novel electric toothbrush head with regular and tapered bristles versus a manual toothbrush for reduction of gingivitis and plaque," International Journal of Dental Hygiene, vol. 17, no. 2, pp. 153-160, 2019.

[7] R. Sabarish, S. R. Chaparala, P. P. Yelisetty, B. Sk, V. Lavu, and M. Mohan, "An in-vitro assessment of the physical and chemical properties of toothbrush bristle following decontamination by three different methods: a pilot study," Cureus, vol. 11, no. 6, p. e4992, 2019.

[8] J. Yaghini, N. Naghsh, S. M. Sadeghi, and S. Soltani, "Gingival inflammatory indices and dental stain index after using aloe vera-green tea mouthwash, matrica mouthwash, or $0.2 \%$ chlorhexidine mouthwash compared with placebo in patients with gingival inflammation," The Open Dentistry Journal, vol. 13, no. 1, 2019.

[9] M. A. Baseer, M. S. Alenazy, M. AlAsqah, M. AlGabbani, and A. Mehkari, "Oral health knowledge, attitude and practices 
among health professionals in King Fahad Medical City," Riyadh. Dental Research Journal, vol. 9, no. 4, p. 386, 2012.

[10] B. Janssens, J. Vanobbergen, M. Lambert, J. M. G. A. Schols, and L. De Visschere, "Effect of an oral healthcare programme on care staff knowledge and attitude regarding oral health: a non-randomised intervention trial," Clinical Oral Investigations, vol. 22, no. 1, pp. 281-292, 2018.

[11] S. Kaur, B. Kaur, and S. S. Ahluwalia, "Oral health knowledge, attitude and practices amongst health professionals in Ludhiana, India," Dentistry, vol. 5, no. 7, p. 1, 2015.

[12] S. Rabiei, S. Z. Mohebbi, R. Yazdani, and J. I. Virtanen, "Primary care nurses' awareness of and willingness to perform children's oral health care," BMC Oral Health, vol. 14, no. 1, p. 26, 2014.

[13] V. Pitchika, C. Pink, H. Völzke, A. Welk, T. Kocher, and B. Holtfreter, "Long-term impact of powered toothbrush on oral health: 11-year cohort study," Journal of Clinical Periodontology, 2019.

[14] R. Sabarish, S. R. Chaparala, B. S. Padma Priya Yelisetty, V. Lavu, and M. Mohan, "An in-vitro assessment of the physical and chemical properties of toothbrush bristle following decontamination by three different methods: a pilot study," Cureus, vol. 11, no. 6, p. e4992, 2019.

[15] S. Pesevska, K. Ivanovski, S. Mindova, A. Kaftandzieva, S. Ristoska, E. Stefanovska et al., "Bacterial contamination of the toothbrushes," Journal of International Dental and Medical Research, vol. 9, no. 1, p. 6, 2016.

[16] M. P. C. Van Leeuwen, F. A. Van der Weijden, D. E. Slot, and M. A. M. Rosema, "Toothbrush wear in relation to toothbrushing effectiveness," International Journal of Dental Hygiene, vol. 17, no. 1, pp. 77-84, 2019.

[17] J.-H. Lee, S.-H. Kim, J.-S. Han, I.-S. L. Yeo, and H.-I. Yoon, "Optical and surface properties of monolithic zirconia after simulated toothbrushing," Materials, vol. 12, no. 7, p. 1158, 2019.

[18] R. Mladenovic, A. Cvetkovic, B. Martinovic et al., "Efficiency of chewable toothbrush in reduction of dental plaque in students," BMC Oral Health, vol. 19, no. 1, p. 58, 2019.

[19] N. Subburaman, P. Madan Kumar, and K. Iyer, "Effectiveness of musical toothbrush on oral debris and gingival bleeding among 6-10-year-old children: a randomized controlled trial," Indian Journal of Dental Research, vol. 30, no. 2, p. 196, 2019.

[20] P. Bhongsatiern, P. Manovilas, M. Songvejkasem, S. Songsiripradubboon, T. Tharapiwattananon, and P. Techalertpaisarn, "Adjunctive use of fluoride rinsing and brush-on gel increased incipient caries-like lesion remineralization compared with fluoride toothpaste alone in situ," Acta Odontologica Scandinavica, vol. 77, pp. 1-7, 2019.

[21] K. Yao, Y. Yao, X. Shen, C. Lu, and Q. Guo, “Assessment of the oral health behavior, knowledge and status among dental and medical undergraduate students: a cross-sectional study," BMC Oral Health, vol. 19, no. 1, p. 26, 2019.

[22] G. Kumar, A. Sethi, R. Tripathi, and s. Pratik, "Assessment of knowledge, attitude, and practice of dental and medical interns toward toothbrush maintenance and replacement in Bhubaneswar city, Odisha, India," Journal of Pharmacy And Bioallied Sciences, vol. 10, no. 2, p. 77, 2018.

[23] D. M. Al Rababah, M. Z. Nassani, O. G. Baker, E. M. Alhelih, S. A. Almomani, and S. Rastam, "Attitudes and practices of nurses toward oral care of hospitalized patients-A crosssectional survey in Riyadh, Saudi Arabia," Journal of Education and Health Promotion, vol. 7, 2018.

[24] A. Alotaibi, S. Alotaibi, M. Alshayiqi, and S. Ramalingam, "Knowledge and attitudes of Saudi intensive care unit nurses regarding oral care delivery to mechanically ventilated patients with the effect of healthcare quality accreditation," Saudi Journal of Anaesthesia, vol. 10, no. 2, p. 208, 2016.

[25] F. Behzadi, S. Khanjari, and H. Haghani, "Impact of an education program on the performance of nurses in providing oral care for mechanically ventilated children," Australian Critical Care, vol. 32, no. 4, pp. 307-313, 2019.

[26] B. Janssens, L. De Visschere, G.-J. van der Putten, K. de LugtLustig, J. M. G. A. Schols, and J. Vanobbergen, "Effect of an oral healthcare protocol in nursing homes on care staffs' knowledge and attitude towards oral health care: a clusterrandomised controlled trial," Gerodontology, vol. 33, no. 2, pp. 275-286, 2016.

[27] N. Al-Yateem, M. Al-Tamimi, M. Brenner et al., "Nurseidentified patient care and health services research priorities in the United Arab Emirates: a Delphi study," BMC Health Services Research, vol. 19, no. 1, p. 77, 2019.

[28] J. Kerr and S. Singh, "Nursing students' attitudes and practices of oral health self-care," African Journal for Physical Activity and Health Sciences (AJPHES), vol. 24, no. 2, pp. 142-154, 2018.

[29] S. da Silva Rodrigues, MdF. C. Caminha, M. G. G. Ferraz, M. A. de Arruda, V. M. da Rocha Kozmhinshy, and C. A. R. de Moraes Guerra, "Knowledge, attitude and practice of the nursing team regarding oral health care in intensive care units in a reference hospital of recife, Brazil," Pesquisa Brasileira em Odontopediatria e Clínica Integrada.vol. 16, no. 1, 2016.

[30] S. Haresaku, H. Aoki, M. Makino, M. Monji, A. Kansui, and K. Kubota, "Practices, attitudes, and confidence of nurses in the performance of Oral health checkups for elderly patients in a Japanese hospital," Oral Health Prev Dent, vol. 16, no. 6, pp. 517-524, 2018.

[31] S. Labeau, D. Vandijck, J. Rello et al., "Evidence-based guidelines for the prevention of ventilator-associated pneumonia: results of a knowledge test among European intensive care nurses," Journal of Hospital Infection, vol. 70, no. 2, pp. 180-185, 2008.

[32] Y. S. Lin, J. C. Chang, T. H. Chang, and M. F. Lou, "Critical care nurses' knowledge, attitudes and practices of oral care for patients with oral endotracheal intubation: a questionnaire survey," Journal of Clinical Nursing, vol. 20, no. 21-22, pp. 3204-3214, 2011.

[33] V. Sreenivasan, A. Ganganna, and P. Rajashekaraiah, "Awareness among intensive care nurses regarding oral care in critically ill patients," Journal of Indian Society of Periodontology, vol. 22, no. 6, p. 541, 2018.

[34] S. Sharif, N. Saddki, and A. Yusoff, "Knowledge and attitude of medical nurses toward oral health and oral health care of pregnant women," The Malaysian Journal of Medical Sciences: MJMS, vol. 23, no. 1, pp. 63-71, 2016.

[35] F. Ahmed, A. Rao, R. Shenoy, and B. S. Suprabha, "Knowledge, attitude, and behavior of nurses toward delivery of primary oral health care in dakshina Kannada, India," Journal of the Indian Society of Pedodontics and Preventive Dentistry, vol. 36, no. 1, pp. 21-25, 2018.

[36] T. Willumsen, L. Karlsen, R. Naess, and S. Bjørntvedt, “Are the barriers to good oral hygiene in nursing homes within the nurses or the patients?" Gerodontology, vol. 29, no. 2, pp. e748-e755, 2012.

[37] E. A. Aikins and J. O. Eigbobo, "Child and maternal oral healthcare: an assessment of the knowledge of nigerian and ghanaian nurses," East African Medical Journal, vol. 91, no. 2, pp. 37-43, 2014. 
[38] J. Gibney, C. Wright, A. Sharma, and V. Naganathan, "Nurses' knowledge, attitudes, and current practice of daily oral hygiene care to patients on acute aged care wards in two Australian hospitals," Special Care in Dentistry, vol. 35, no. 6, pp. 285-293, 2015.

[39] M. Janatolmakan, S. Kakazadeh, B. Andayeshgar, F. Jafari, and A. Khatony, "Comparison of knowledge, attitude, and practice of nursing and medical students in Kermanshah, Iran, about toothbrush maintenance and use," Journal of Environmental and Public Health, p. 2021, 2021.

[40] H. Frenkel, I. Harvey, and K. Needs, "Oral health care education and its effect on caregivers' knowledge and attitudes: a randomised controlled trial," Community Dentistry and Oral Epidemiology, vol. 30, no. 2, pp. 91-100, 2002. 